\title{
Smart Management Energy Systems in Industry 4.0
}

\author{
Renato Ferrero $^{1, * \mathbb{C}}$, Mario Collotta ${ }^{2}$, Maria Victoria Bueno-Delgado ${ }^{3} \mathbb{( i )}$ and \\ Hsing-Chung Chen 4 \\ 1 Dipartimento di Automatica e Informatica, Politecnico di Torino and Italy, 10129 Torino TO, Italy \\ 2 Faculty of Engineering and Architecture, Kore University of Enna, 94100 Enna EN, Italy; \\ mario.collotta@unikore.it \\ 3 Department of Information Technologies and Communications, Technical University of Cartagena, \\ 30202 Cartagena, Spain; mvictoria.bueno@upct.es \\ 4 Department of Computer Science and Information Engineering, Asia University, Taichung City 41354, \\ Taiwan; cdma2000@asia.edu.tw \\ * Correspondence: renato.ferrero@polito.it
}

Received: 10 December 2019; Accepted: 1 January 2020; Published: 13 January 2020

\section{Overview}

In its origins, the term Industry 4.0 was associated with the computerization of manufacturing and with the diffusion of new network technologies in order to improve the communication paradigm. Today, the definition and implementation of Industry 4.0 include a number of trends, such as the Internet of Things (IoT), digital manufacturing and cyber-physical systems. Among these key elements, energy aware systems in factory automation are emerging as a challenging trend of Industry 4.0. Technological advancements in the ability to collect, transfer and analyze data by using smart energy aware systems are at the aim of this trend.

Smart solutions in order to limit the power consumption of manufacturing line aim at developing and integrating new technologies and methods into smart factories in order to rapidly adapt and respond to changes in the markets' demands for high-quality products. In fact, smart energy aware systems in factories lie at the core of both Industry 4.0 and smart manufacturing.

A variety of recent advanced technologies and approaches play important roles, by exploiting innovative technologies and solutions and/or optimization methods. They allow higher levels of adaptively and flexibility in energy aware systems.

This Special Issue of Energies on the subject area of "Smart Management Energy Systems in Industry 4.0 " has solicited high quality and unpublished work on recent advances in energy aware smart systems in Industry 4.0. Several researchers have replied to our call, with the following statistics:

- $\quad$ Submissions (17);

- Publications (5);

- $\quad$ Rejections (12);

The following paragraph summarizes the main topics of the papers published in the Special Issue [1-5]. Authors' geographical distribution of published papers is: 2 from Taiwan, 2 from China, and 1 from Spain.

\section{Summary of the Special Issue}

We found the edition and selections of papers for the Special Issue inspiring and rewarding. We also thank the editorial staff and reviewers for their efforts and help during the process.

The authors in [1] show a real-time pricing scheme in order to connect one energy provider (response) and multiple energy hub operators (demand). The paper, by using the Stackelberg game 
approach, shows the interactions among the energy provider (leader) and energy consumers (follower). A distributed algorithm was proposed, in order to achieve the best strategies for the energy provider and each energy hub operator. Benefits of the described method, shown through simulation scenarios, regard balancing of energy supply and demand.

The paper [2] is focused on the optimization of power consumption in public buildings in China. This aim could be obtained by following several parameters such as: prediction of natural illuminance, outdoor air temperature and flow of people in public building. Despite the unpredictability of these parameters, prediction error introduced by conventionally approaches can be exploited in order to produce a strategy that allows to reduce the power consumption taking into account the forecasting uncertainty of environmental factors.

The analysis establishes some power consumption models for three electrical systems, where it is crucial to develop power-consumption aware solutions: lighting systems, air-conditioning systems, and elevator systems in public buildings. For each system, some robust indexes are established, in order to formulate the objective function that allows to reduce the total electricity cost.

The authors in [3] focused their work in smart living, where indoor positioning location techniques are commonly used for performing smart home power management. In this work, the study proposes a novel system framework focused on Bluetooth Low Energy (BLE). The key is to detect user location in order to perform power management in the home through a mobile device application. Since the BLE beacon may produce a multipath effect, the study implements a positioning algorithm and hardware configuration to reduce the error rate.

The study also includes location fingerprint positioning algorithm and filter modification to set a positioning method, in order to make easier the deployment, as well as to reduce the required computing resources. The authors evaluated the Received Signal Strength Indicators (RSSI) and different filters, opening a discussion about the relationship between the signal accuracy of BLE beacon and the number of BLE beacons. The BLE beacon multilateration positioning was also combined with the In-Snergy intelligent energy management system for smart home power management.

The paper [4] presents a novel technique to optimize robotized manufacturing lines in terms of energy consumption. The goal is to reduce or eliminate the idle time that robots spend to calibrate velocity. The technique is focused on an idle-time predicting algorithm for each next cycle, based on Miniterm 4.0. This concept is defined as the part of the machine that can be replaced in an easier way than any other part by the maintenance workers, focused on the Industry 4.0 adoption.

The predictive control technique measures in real time the sub-cycle times (miniterms) with the goal to detect disturbances that predict future machine failures. The idle time is computed through the miniterms and the velocity for the robot is adjusted. The technique is tested in a real production line in Spain. Finally, an interesting discussion about the limitations in the technique and further implementations ends the paper.

The authors in [5] focus their research on the energy consumption caused by thousands of sensors spread over smart factories. Sensors collect data of interest (for monitoring manufacturing processes, objects, machinery and workers) in real time and send them to data centers, which process them and react consequently in order to achieve an efficient management and decision-making. Data compression is the key for reducing data size and then, energy consumption in the transmission and storage. In general, compression ratios of lossless compression approaches are not high.

Literature shows that Bounded-error approximation to the original sensor data can retain a certain level of quality of the compressed data. Many Bounded-Error Piecewise Linear Approximation (BEPLA) methods have been proposed, focused on how to approximate to the original data with fewer line segments. The resolution reduction when creating BEPLA is adopted in [5]. On selecting end point for BEPLA, resolution reduction sets a new restriction so that fewer bits are allowed to encode the end points of line segments. A variant method with Swing filter technique is proposed, called Swing-RR. Experimental results show that the novel technique has optimal time and space complexities in processing data records. In real datasets, the experiment results show that Swing-RR 
uses more line segments than a set of state-of-the-art methods evaluated, and the size of sensor data are significantly reduced by resolution reduction, implying that the mean square errors between the original data and compressed data are also smaller. Consequently, the energy consumed for delivering and saving compressed data is significantly lowered.

\section{References}

1. Ma, T.; Wu, J.; Hao, L.; Yan, H.; Li, D. A Real-Time Pricing Scheme for Energy Management in Integrated Energy Systems: A Stackelberg Game Approach. Energies 2018, 11, 2858. [CrossRef]

2. Xiao, J.; Xie, J.; Chen, X.; Yu, K.; Chen, Z.; Luan, K. Robust Optimization of Power Consumption for Public Buildings Considering Forecasting Uncertainty of Environmental Factors. Energies 2018, 11, 3075. [CrossRef]

3. Ke, C.; Wu, M.; Chan, Y.; Lu, K. Developing a BLE Beacon-Based Location System Using Location Fingerprint Positioning for Smart Home Power Management. Energies 2018, 11, 3464. [CrossRef]

4. Garcia, E.; Montés, N. Real-Time Idle Time Cancellation by Means of Miniterm 4.0. Energies 2019, 12, 1230. [CrossRef]

5. Lin, J.-W.; Liao, S.-W.; Leu, F.-Y. Sensor Data Compression Using Bounded Error Piecewise Linear Approximation with Resolution Reduction. Energies 2019, 12, 2523. [CrossRef]

(C) 2020 by the authors. Licensee MDPI, Basel, Switzerland. This article is an open access article distributed under the terms and conditions of the Creative Commons Attribution (CC BY) license (http://creativecommons.org/licenses/by/4.0/). 